\title{
Baryon asymmetry, dark matter and neutrino mass via exotic multiplets
}

\author{
Sandy S. C. Law* \\ Department of Physics, \\ National Cheng-Kung University, \\ Tainan 701, Taiwan \\ E-mail: slaw@mail.ncku.edu.tw
}

\begin{abstract}
We demonstrate that by adding three exotic fermion 5-plets and one scalar 6-plet to the standard model, a consistent solution to the problems of baryon asymmetry, dark matter and neutrino mass can be obtained. This is possible because renormalizability and standard model gauge invariance allow the lightest exotic particle in this model to be a dark matter candidate if the new 6-plet scalar does not develop a nonzero vacuum expectation value. Furthermore, light neutrino masses can be generated radiatively at one-loop while the baryon asymmetry is produced by the CP-violating decays of the second lightest exotic particle.
\end{abstract}

36th International Conference on High Energy Physics,

July 4-11, 2012

Melbourne, Australia

* Speaker. 


\section{Introduction}

It is clear that some kind of extension of the standard model (SM) is required in order to explain the observed cosmic baryon asymmetry, effects of dark matter (DM) or nonzero neutrino masses. While there are numerous ways to add new physics to the SM, in this work, we explore the possibility of solving these problems via the introduction of several exotic $\mathrm{SU}(2)_{L}$ multiplets [1] (see also [2,3]). This setup is motivated by the ideas of minimal DM [4] (where renormalizability and SM gauge invariance are enough to ensure the DM stability), and the possible link between WIMP-like DM and neutrino masses via radiative diagrams [5]. With these ideas, we aim to build an exotic multiplet model which can simultaneously solve the problems of DM, neutrino mass, as well as the baryon asymmetry of the universe. Subsequently, we wish to understand the parameter space (and fine-tunings, if any) required for a consistent solution.

\section{The model}

In order to construct a consistent solution to baryon asymmetry, dark matter and neutrino masses, we extend the particle content of the minimal SM by adding three fermion multiplets, $N_{k},(k=1,2,3)$, and one scalar multiplet, $\chi$, with SM gauge transformation properties:

$$
N_{k} \sim(1,5,0), \quad \text { and } \quad \chi \sim(1,6,-1 / 2)
$$

respectively. This choice is dictated by the "minimal DM" idea, as well as our demand for the lightest fermion 5-plet to be the DM candidate. Other smaller multiplet combinations for these new particles will result in unwanted terms in the scalar potential which can destabilize our DM candidate, or give phenomenologically unacceptable component fields that have fractional charges. ${ }^{1}$ The Lagrangian of interest for this model is given by

$$
\mathscr{L}_{\text {int }}=i \bar{N}_{k} \not \supset N_{k}+\left(D^{\mu} \chi\right)^{\dagger}\left(D_{\mu} \chi\right)-\left[h_{j k} \bar{L}_{j} \chi N_{k}+\frac{1}{2} \overline{\left(N_{k}\right)^{c}} M_{k} N_{k}+\text { h.c. }\right]-V_{S},
$$

where $L_{j}=(v, \ell)_{j}^{T}$ is the $j$-flavor LH lepton doublet, $D_{\mu}$ denoting the SM covariant derivative, and $h_{j k}$ is the $(j, k)$-element of the Yukawa coupling matrix which is assumed to be complex. If we assume the (technically natural) limit $\chi^{\dagger} \chi \chi \phi \ll 1$, then the scalar potential $V_{S}$ is given by

$$
V_{S}=\mu_{\phi}^{2} \phi^{\dagger} \phi+\mu_{\chi}^{2} \chi^{\dagger} \chi+\frac{\lambda_{\phi}}{2}\left(\phi^{\dagger} \phi\right)^{2}+\frac{\lambda_{\chi \alpha}}{2}\left(\chi^{\dagger} \chi\right)_{\alpha}^{2}+\lambda_{\phi \chi \beta}\left(\phi^{\dagger} \phi \chi^{\dagger} \chi\right)_{\beta}+\frac{1}{2}\left[\lambda_{\phi \chi}^{\prime}(\phi \chi)^{2}+\text { h.c. }\right],
$$

with $\phi=\left(\phi^{+}, \phi^{0}\right)^{T}$ being the SM Higgs doublet. The subscripts $\alpha$ and $\beta$ in (2.3) denote the many independent ways to contract the components of the $\mathrm{SU}(2)_{L}$ multiplets involved, and we sum over them (see $[1,2])$. To ensure that the neutral component of the lightest exotic particle can be the DM candidate (assumed to be $N_{1}$ ), the parameters in $V_{S}$ must be such that scalar 6-plet $\chi$ will not develop a nonzero vacuum expectation value (i.e. $\langle\chi\rangle=0$ ). Together with $\chi^{\dagger} \chi \chi \phi \ll 1$, these are the key fine-tuning conditions coming from the scalar sector.

\footnotetext{
${ }^{1}$ The choice of using a 6-plet scalar is purely a model building decision since, strictly speaking, a 4-plet scalar is also workable.
} 
Once these are satisfied, the choices for the exotic mass spectrum $\left(M_{1,2,3}\right.$ and $\left.M_{\chi}\right)$, the Yukawas $\left(h_{j k}\right)$ and the quartic coupling $\left(\lambda_{\phi \chi}^{\prime}\right)$ will determine the solution to baryogenesis, neutrino mass and DM. In particular, if we demand that $M_{1} \ll M_{\chi} \ll M_{2} \ll M_{3}$, then $N_{1}$ will be the DM candidate while baryogenesis via leptogenesis can be achieved through the CP asymmetric decays of the second lightest exotics $\chi$. Finally, neutrinos will gain a mass via one-loop diagrams in this model.

\section{The dark matter candidate}

In our model, with $N_{1}^{0}$ being the lightest among all the new exotic states and given that Lagrangian (2.2) possesses a $Z_{2}$ symmetry $\left(\psi_{\mathrm{SM}} \rightarrow \psi_{\mathrm{SM}}\right.$ and $\left.N_{k}, \chi \rightarrow-N_{k},-\chi\right)$, it is the prime DM candidate. Like in other WIMP models, the ability to reproduce the observed DM relic density is a crucial ingredient in determining whether the DM candidate under investigation is a viable one. As a result, we assume that the standard thermal freeze out of these $N_{1}^{0}$ 's are solely responsible for the observed DM relic abundance. Furthermore, we shall assume that interactions mediated by SM gauge bosons will dominate the (co)annihilations cross-section relevant for DM relic calculation.

Following the canonical method for computing the relic density and using the cross-section derived from the kinetic Lagrangian in (2.2)

$$
\begin{aligned}
\sqrt{10} \mathscr{L}_{\text {kin }}= & \sqrt{2}\left(g \cos \theta_{w} Z_{\mu}-e A_{\mu}\right)\left(2 \overline{N_{1}^{++}} \gamma^{\mu} N_{1}^{++}+\overline{N_{1}^{+}} \gamma^{\mu} N_{1}^{+}-\overline{N_{1}^{-}} \gamma^{\mu} N_{1}^{-}-2 \overline{N_{1}^{--}} \gamma^{\mu} N_{1}^{--}\right) \\
& +g\left[W_{\mu}^{+}\left(\overline{N_{1}^{++}} \gamma^{\mu} N_{1}^{+}+\overline{N_{1}^{+}} \gamma^{\mu} N_{1}^{0}+\overline{N_{1}^{0}} \gamma^{\mu} N_{1}^{-}+\overline{N_{1}^{-}} \gamma^{\mu} N_{1}^{--}\right)+\text {h.c. }\right], e>0,
\end{aligned}
$$

it is estimated that for a $N_{1}$ mass of

$$
M_{1} \gtrsim 10 \mathrm{TeV},
$$

the observed DM abundance can be explained [4]. This result is in line with the typical WIMP type models where $\mathrm{TeV}$ scale DM are predicted.

\section{Baryogenesis via $N_{2}$-leptogenesis}

The scenario of thermal leptogenesis [6] is a very attractive solution for the baryogenesis problem for it also contains the right elements for generating nonzero neutrino masses. While the traditional leptogenesis scenario relies on the $\mathrm{CP}$ (and lepton number) violating decays of the lightest new singlet state (e.g. $N_{1}$ ) in the model to create the baryon asymmetry, non-standard cases where the second lightest state (e.g. $N_{2}$ ) playing the major role may also be workable under certain circumstances [7, 8]. In general, one must take flavor effects [7, 8, 9] into account if $N_{2}$-leptogenesis is the dominating asymmetry generating process.

In our exotic multiplet model, the Yukawa terms in (2.2), $h_{j k} \bar{L}_{j} \chi N_{k}$ for $k \neq 1$ can give rise to the lepton number violating process: $N_{k \neq 1} \rightarrow L_{j} \chi^{\dagger}$ in the early universe (see Fig. 1). The (incoherent) sum of all contributions in each component gives rise to the tree-level decay rate:

$$
\Gamma\left(N_{k} \rightarrow L_{j} \chi^{\dagger}\right) \equiv \Gamma\left(\bar{N}_{k} \rightarrow \bar{L}_{j} \chi\right)=\frac{h_{j k}^{*} h_{j k}}{16 \pi} M_{k}\left(1-\frac{M_{\chi}^{2}}{M_{k}^{2}}\right)^{2}, \quad k \neq 1
$$


(a)

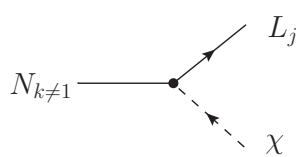

(b)

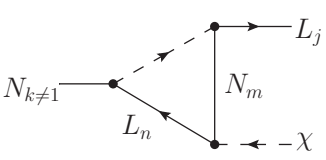

(c)

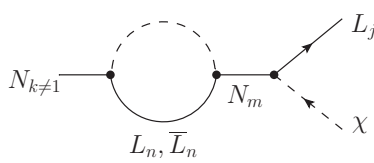

Figure 1: The lepton number violating (a) tree-level, (b) one-loop vertex and (c) one-loop self-energy diagrams for $N_{k}$ multiplet decays $(k \neq 1)$. For $k=1$, these processes are kinematically forbidden.

The corresponding CP asymmetry for this decay is defined as

$$
\varepsilon_{k j} \equiv \frac{\Gamma\left(N_{k} \rightarrow L_{j} \chi^{\dagger}\right)-\Gamma\left(\bar{N}_{k} \rightarrow \bar{L}_{j} \chi\right)}{\sum_{j} \Gamma\left(N_{k} \rightarrow L_{j} \chi^{\dagger}\right)+\sum_{j} \Gamma\left(\bar{N}_{k} \rightarrow \bar{L}_{j} \chi\right)},
$$

with the leading nonzero contribution coming from the interference terms between the tree and oneloop diagrams (see Fig. 1). Applying the DM constraint of (3.2) and assuming the mass spectrum

$$
M_{3} \gg 10^{12} \mathrm{GeV}, \quad 10^{12} \mathrm{GeV} \gg M_{2} \gg 10^{9} \mathrm{GeV}, \quad 10^{9} \mathrm{GeV} \gg M_{\chi} \gg M_{1} \simeq 10^{4} \mathrm{GeV},
$$

this scenario is qualitatively very similar to the "standard" $N_{2}$-leptogenesis with hierarchical $N_{k}$ 's. ${ }^{2}$

In the light of this, the analysis is quite standard and much of the knowledge gained in the canonical scenario may be reapplied here. One important thing to note is that unlike $N_{1}$ leptogenesis, the $N_{2}$ version requires tracking the evolution of the lepton asymmetry through two distinct stages: asymmetry production at $T \sim M_{2}$ and subsequent washout at $T \sim M_{\chi}$ [1]. Consequently, some additional assumptions are needed regarding how to handle the transition period between the two stages.

However, assuming that those subtleties can be managed in a systematic way, the rest of the analysis is actually quite straightforward. As in standard leptogenesis with flavor effects, one defines the flavor projectors, which has a tree-level realization as $P_{2 j}^{0} \equiv \bar{P}_{2 j}^{0}=h_{j 2}^{*} h_{j 2} /\left(h^{\dagger} h\right)_{22}$, where $h$ is the Yukawa coupling in (2.2). With this, we may write down the set of evolution equations for $N_{2}$ and lepton asymmetry $\Delta_{j}$ during the production stage as

$$
\begin{gathered}
\frac{d \mathscr{N}_{N_{2}}}{d z}=-D_{2}\left(\mathscr{N}_{N_{2}}-\mathscr{N}_{N_{2}}^{\mathrm{eq}}\right), \\
\frac{d \mathscr{N}_{\perp}}{d z}=-\varepsilon_{2 \perp} D_{2}\left(\mathscr{N}_{N_{2}}-\mathscr{N}_{N_{2}}^{\mathrm{eq}}\right)-P_{2 \perp}^{0} W_{2} \sum_{j=\perp, \tau} C_{\perp j}^{f=2} \mathscr{N}_{\Delta_{\perp}}, \\
\frac{d \mathscr{N}_{\Delta \tau}}{d z}=-\varepsilon_{2 \tau} D_{2}\left(\mathscr{N}_{N_{2}}-\mathscr{N}_{N_{2}}^{\mathrm{eq}}\right)-P_{2 \tau}^{0} W_{2} \sum_{j=\perp, \tau} C_{\tau j}^{f=2} \mathscr{N}_{\Delta_{\tau}},
\end{gathered}
$$

where $z=M_{2} / T . D_{2}$ and $W_{2}$ denote the decay and washout terms respectively. The fact that the flavor coupling matrix $C^{f=2}$ is non-diagonal ensures that the differential equations are coupled, and signifying the importance of flavor effects in the evolution. Similarly, one can write down the equations for the washout stage as above except that the $\varepsilon_{2 j}$ term will be absent and the system will be generalized to the three-flavor regime. These systems of equations may then be solved using the standard techniques.

In order to ensure successful $N_{2}$-leptogenesis, it turns out that the total asymmetry should originate mainly from decays in the tau flavor [7,8]. This implies that the flavored decay parameters (e.g. $K_{2 \tau}$ ), ${ }^{3}$ which are basically functions of the flavor projectors (e.g. $P_{2 \tau}^{0}$ ), are constrained. In

\footnotetext{
${ }^{2}$ In the standard case, " $N_{k}$ " is a $\mathrm{SU}(2)_{L}$ singlet rather than a 5-plet.

${ }^{3}$ See [1] for the precise definitions.
} 


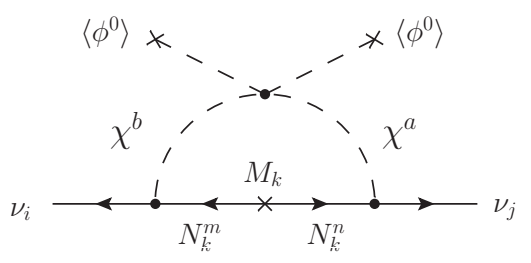

Figure 2: The one-loop diagram for generating light neutrino mass term $\left(M_{V}\right)_{i j}$.

particular, one typically requires

$$
K_{2 \tau} \gtrsim 1 \quad \text { and } \quad K_{\chi \tau} \ll 1
$$

\section{Neutrino mass}

In this model, because $\langle\chi\rangle=0$, there is no Dirac mass term for the light neutrino. However, nonzero neutrino masses can be generated at one-loop level (see Fig. 2). After expanding out the components and summing all contributions, one finds that the radiative diagrams will lead to the light neutrino mass matrix

$$
\left(M_{v}\right)_{i j}=\frac{7}{5760 \pi^{2}} \lambda_{\phi \chi}^{\prime}\left\langle\phi^{0}\right\rangle^{2} \sum_{k} \frac{h_{i k} h_{j k}}{M_{k}} \frac{y_{k}}{1-y_{k}}\left[1+\frac{y_{k} \ln y_{k}}{1-y_{k}}\right], \quad \text { where } y_{k} \equiv \frac{M_{k}^{2}}{M_{\chi}^{2}} .
$$

The individual light neutrino mass is obtained by diagonalizing $M_{v}$ with the neutrino mixing matrix, $U_{\mathrm{PMNS}}$ :

$$
m_{n}^{v}=\sum_{i, j}\left(U_{\mathrm{PMNS}}^{\dagger}\right)_{n i}\left(M_{v}\right)_{i j}\left(U_{\mathrm{PMNS}}^{*}\right)_{j n}, \quad n=1,2,3
$$

\section{A sample solution}

As an existence proof, we present a set of parameters which satisfies the requirements from all three sectors (i.e. Eqs. (3.2), (4.7) and (5.2)). Firstly, we set the exotic masses to be

$$
M_{1} \simeq 10^{4} \mathrm{GeV}, \quad M_{\chi} \simeq 10^{7} \mathrm{GeV}, \quad M_{2} \simeq 10^{10} \mathrm{GeV}, \quad M_{3} \simeq 10^{13} \mathrm{GeV},
$$

where $M_{1}$ is specifically chosen to satisfy (3.2). For the light neutrino sector, we appeal to the best fit values from oscillation experiments [10],

$$
\begin{aligned}
& \Delta m_{12}^{2} \approx 7.59 \times 10^{-5} \mathrm{eV}^{2}, \quad\left|\Delta m_{23}^{2}\right| \approx 2.40 \times 10^{-3} \mathrm{eV}^{2}, \\
& \sin ^{2} \theta_{12} \approx 0.318, \quad \sin ^{2} \theta_{23} \approx 0.50, \quad \sin ^{2} \theta_{13} \approx 0.013 .
\end{aligned}
$$

Let assume, for illustration purpose, that the lightest LH neutrino has mass $m_{1} \approx 0.002 \mathrm{eV}$ and the spectrum is normal hierarchical. Moreover, for simplicity, we ignore the phases in the $U_{\mathrm{PMNS}}$ matrix. To satisfy the neutrino and leptogenesis constraints, we pick respectively

$$
\lambda_{\phi \chi}^{\prime}=0.1, K_{2 \tau} \simeq 65, K_{\chi \tau} \simeq 0.1 .
$$


The rest of the $h_{j k}$ entries may now be solved simultaneously (using the neutrino data), and one possible set of solutions for the complex Yukawas is

$$
\begin{gathered}
h_{e 1}=1.23+0.359 i, \quad h_{e 2}=0.104-0.329 i, \quad h_{e 3}=-0.344+0.263 i, \\
h_{\mu 1}=1.71-1.02 i, \quad h_{\mu 2}=-0.304-0.468 i, \quad h_{\mu 3}=-3.76+0.367 i, \\
h_{\tau 1}=1.07 \times 10^{-5}, \quad h_{\tau 2}=8.88 \times 10^{-3}, \quad h_{\tau 3}=5.34 .
\end{gathered}
$$

Using these input values, one finds that $\eta_{B} \simeq 6.3 \times 10^{-10}$ which agrees with the CMB data.

Hence, we have demonstrated that by introducing three exotic fermion 5-plets and one scalar 6-plet to the SM, a consistent solution to the problems of baryon asymmetry, dark matter and neutrino mass can be obtained.

\section{Acknowledgments}

This work is supported in part by the NSC (grant numbers: NSC-100-2112-M-006-014-MY3 and NSC-100-2811-M-006-019) and in part by the NCTS of Taiwan.

\section{References}

[1] C. -H. Chen and S. S. C. Law, Phys. Rev. D 85, 055012 (2012) [arXiv:1111.5462 [hep-ph]].

[2] Y. Cai, X. -G. He, M. Ramsey-Musolf and L. -H. Tsai, JHEP 1112, 054 (2011) [arXiv:1108.0969 [hep-ph]].

[3] K. Kumericki, I. Picek and B. Radovcic, JHEP 1207, 039 (2012) [arXiv:1204.6597 [hep-ph]].

[4] M. Cirelli, N. Fornengo, A. Strumia, Nucl. Phys. B753, 178-194 (2006). [hep-ph/0512090]; M. Cirelli, A. Strumia, M. Tamburini, Nucl. Phys. B787, 152-175 (2007). [arXiv:0706.4071 [hep-ph]]; M. Cirelli, A. Strumia, New J. Phys. 11, 105005 (2009). [arXiv:0903.3381 [hep-ph]].

[5] L. M. Krauss, S. Nasri and M. Trodden, Phys. Rev. D 67, 085002 (2003) [hep-ph/0210389]; E. Ma, Phys. Rev. D 73, 077301 (2006) [arXiv:hep-ph/0601225].

[6] M. Fukugita, T. Yanagida, Phys. Lett. B174, 45 (1986).

[7] S. Antusch, P. Di Bari, D. A. Jones and S. F. King, Nucl. Phys. B 856, 180 (2012) [arXiv:1003.5132 [hep-ph]].

[8] E. Bertuzzo, P. Di Bari and L. Marzola, Nucl. Phys. B 849, 521 (2011) [arXiv:1007.1641 [hep-ph]].

[9] O. Vives, Phys. Rev. D 73, 073006 (2006) [arXiv:hep-ph/0512160]; E. Nardi, Y. Nir, E. Roulet and J. Racker, JHEP 0601, 164 (2006) [arXiv:hep-ph/0601084]; R. Barbieri, P. Creminelli, A. Strumia, N. Tetradis, Nucl. Phys. B575, 61-77 (2000). [hep-ph/9911315]; A. Abada, S. Davidson, F. X. Josse-Michaux, M. Losada and A. Riotto, JCAP 0604, 004 (2006) [arXiv:hep-ph/0601083]; A. Abada, S. Davidson, A. Ibarra, F. X. Josse-Michaux, M. Losada and A. Riotto, JHEP 0609, 010 (2006) [arXiv:hep-ph/0605281]; S. Blanchet and P. Di Bari, JCAP 0703, 018 (2007) [arXiv:hep-ph/0607330]; F. X. Josse-Michaux and A. Abada, JCAP 0710, 009 (2007) [arXiv:hep-ph/0703084].

[10] T. Schwetz, M. A. Tortola and J. W. F. Valle, New J. Phys. 10, 113011 (2008) [arXiv:0808.2016 [hep-ph]]. 